\title{
Calculation of Carrier Distribution, Space Charge Accumulation, and Internal Electric Field Distribution in CdZnTe Detectors under Extremely High X-Ray Doses
}

\author{
Xiangxiang Luo, Zhuotong Du, Gangqiang Zha, Lingyan Xu and Wanqi Jie* \\ State Key Laboratory of Solidification Processing, China \\ *Corresponding author: Wanqi Jie, State Key Laboratory of Solidification Processing, and MIIT Key Laboratory of Radiation Detection Materials and \\ Devices, China
}

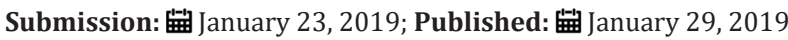

\begin{abstract}
We calculated the carrier density, space charge and internal electric field distribution in CdZnTe planar detectors with the consideration of four kinds of defect traps to understand its detection properties under ultrahigh X-ray flux. The results showed that severe space charge collection occurred, and the internal electric field was distorted when the X-ray flux was ultrahigh. This could strongly influence detector performance.
\end{abstract}

Keywords: Carrier distribution; X-ray detector; CdZnTe crystal

PACS: 66.30.J-, 72.20.Jv, 71.55.Gs

\section{Introduction}

Photon counting CdZnTe (or Cadmium Zinc Telluride) detectors have been accepted as a new generation device for X-ray imaging in the last decades [1]. In this case, CdZnTe detector may work under extremely high X-ray dose [2-4]. For example, in medical CT, $\mathrm{X}$-ray flux can be as high as $10^{8}$ Photon $/ \mathrm{s} / \mathrm{mm}^{2}$ to obtain images with enough contrast [5-7]. However, CdZnTe crystal was found to have polarization effect under such high doses of X-ray $[7,8]$ On the other side, the amplitude of the charge signal generated by a detector can be extremely low, even at the same level of noise $[9,10]$. In this case, the detector will be in a "stagnation" state and cannot work properly [11-13]. Therefore, the application requires $\mathrm{X}$-ray detector to possess high resolution at low X-ray dose as well as to bear high X-ray flux without deterioration. Such a task of CZT X-ray detectors is usually hindered by the existence of highdensity non-equilibrium carriers in the crystal, particularly holes with a low mobility lifetime, which are trapped by deep level defects [14-16]. The electrostatic repulsion force of the crystal defects accumulates space charge. When the accumulated space charge reaches a certain value, the electric field will be distorted, which will severely impede the transport process of carriers and deteriorate the performance of the detector [17-19]. In the present paper, the space charge accumulation behaviors and their effects on the detector performance will be evaluated by analyzing the trap and de-trap process of X-ray stimulated charges.

\section{Methods and Calculations}

The theoretical method was proposed for the calculations of point defect effects, particularly deep-level point defects, on the space charge accumulation under extremely high doses of X-ray radiation. We used a planar detector as an example to simplify the calculation of space charge accumulation. It is assumed that $\mathrm{E}_{\mathrm{A}[1 \sim \mathrm{m}]}$ and $\mathrm{E}_{\mathrm{D}[1 \sim \mathrm{m}]}$ are the acceptor levels and donor levels in the energy band structure of CdZnTe crystal, $\mathrm{N}_{\mathrm{T}[1 \sim \mathrm{m}]}$ and $\mathrm{P}_{\mathrm{T}[1 \sim \mathrm{m}]}$ are the defect concentrations and $\sigma_{\mathrm{A}[1 \sim \mathrm{m}]}$ and $\sigma_{\mathrm{D}[1 \sim \mathrm{m}]}$ are the captured cross sections, $\mathrm{p}$ and $\mathrm{n}$ are the concentrations of holes and free electrons, and are the concentrations of the trapped electron and hole in the $\mathrm{m}^{\text {th }}$ defect level, respectively. The capture rate of electrons at the $\mathrm{m}^{\text {th }}$ donor level is

$$
W_{e t}^{m}=\sigma_{D m} V\left(N_{T m}-n_{t}^{m}\right)
$$

The de-trapping rate is

$$
W_{e d}^{m}=N_{c} \sigma_{D m} V \exp \left(-\left(E_{c}-E_{D m}\right) / k T\right)
$$

where $\mathrm{V}$ is the average rate of carrier thermal motion.

Corresponding to the holes, the trapping rate and de-trapping rate at the $\mathrm{m}^{\text {th }}$ acceptor level are

$$
W_{h t}^{m}=\sigma_{A m} V\left(P_{T m}-p_{t}^{m}\right)
$$




$$
W_{h d}^{m}=N_{v A m} V \exp \left(-\left(E_{A m}-E_{V}\right) / k T\right)
$$

where $\mathrm{N}_{v}$ is the effective density of states in the valence band.

The incidence of the complex action of the carriers trapped at the $\mathrm{m}^{\text {th }}$ defect level is

$$
\text { Electrons: } U_{e r}^{m}=\sigma_{e r}^{m} V p_{t}^{m}
$$

Holes: $U_{h r}^{m}=\sigma_{h r}^{m} V n_{t}^{m}$

where $\sigma_{e r}^{m}$ and $\sigma_{h r}^{m}$ are the composite cross sections of electrons and holes, respectively.

Considering extremely high dose irradiation, there will be a large flux of non-equilibrium carriers in CdZnTe crystal. In this case, the probability of direct electron recombination in the conduction band and valence band will be significantly increased. $\mathrm{R}$ represents the unit volume per unit time. The flux is directly compounded.

$$
R=\sigma_{R} V\left(n p-n_{o} p_{o}\right)
$$

where $\sigma_{\mathrm{R}}$ is the direct composite cross-section area, and $\mathrm{n}_{0}$ and $\mathrm{p}_{0}$ are the concentrations of free electrons and holes at thermal equilibrium, respectively.

It is considered that external bias is sufficiently high to neglect the diffusion motion of carriers. Based on the carrier continuity equation and the law of conservation of charge, we can derive the relationship between carrier concentration, time $t$, and spatial dimension $\mathrm{Z}$ (the external electric field along $\mathrm{Z}$ direction).

$$
\begin{aligned}
& \frac{\partial n}{\partial t}=-n \mu_{e} \frac{\partial E}{\partial Z}-\mu_{e} E \frac{\partial n}{\partial z}-\sum_{k=1}^{m}\left(W_{e t}^{k} n-W_{e d}^{k} n_{t}^{k}\right)-\sum_{k=1}^{m} U_{h r}^{m}-R+f \\
& \frac{\partial p}{\partial t}=p \mu_{h} \frac{\partial E}{\partial Z}+\mu_{h} E \frac{\partial p}{\partial z}-\sum_{k=1}^{m}\left(W_{h t}^{k} p-W_{h d}^{k} p_{t}^{k}\right)-\sum_{k=1}^{m} U_{e r}^{m}-R+f
\end{aligned}
$$

$$
\begin{aligned}
\frac{\partial n_{t}^{k}}{\partial t} & =W_{e t}^{k} n-W_{e d}^{k} n_{t}^{k}+U_{e d r}^{k}\left(N_{T k}-n_{t}^{k}\right)-U_{e r}^{k} p \\
\frac{\partial p_{t}^{k}}{\partial t} & =W_{h t}^{k} p-W_{h d}^{k} p_{t}^{k}+U_{h d r}^{k}\left(P_{T k}-p_{t}^{k}\right)-U_{h r}^{k} n
\end{aligned}
$$

where $\mu_{\mathrm{e}}$ and $\mu_{\mathrm{h}}$ are the mobility of electron and hole respectively, $f$ is the incidence of electron-hole pairs determined by the incident ray, and $\mathrm{E}$ is the magnitude of the electric field generated by the external bias in the crystal. From $E(Z, t)=-\partial \varphi / \partial Z$ and Poisson's equation, we have

$$
\frac{d^{2} \varphi}{d Z^{2}}=\frac{q}{\varepsilon}\left(p-\sum_{k=1}^{m}\left(P_{T k}-p_{t}^{k}\right)-n+\sum_{k=1}^{m}\left(N_{T k}-n_{t}^{k}\right)\right)
$$

The boundary conditions are

$$
\varphi(0)=0 ; \varphi(d)=V
$$

where $\mathrm{d}$ is the thickness of the detector and $\varepsilon$ is the dielectric constant of CdZnTe.

We believe that X-ray energy is evenly distributed with the average value at the half of the applied voltage. According to the absorption rate of different energy rays in different CdZnTe crystals, we can determine how the rays are deposited in a crystal. The total energy generated by X-rays is

$$
E_{x}=V_{x} I_{x} \eta A
$$

where $V_{x}$ is the ray tube voltage, $I_{x}$ is the tube current of the ray machine, and $\eta$ is the power coefficient, which is related to the size of the tube voltage. A is the acceptance coefficient, which is related to the distance between the crystal and ray source. To solve partial differential equations (8) and (9), we use a finite element to create m CdZnTe crystal elements, as shown in Figure 1.

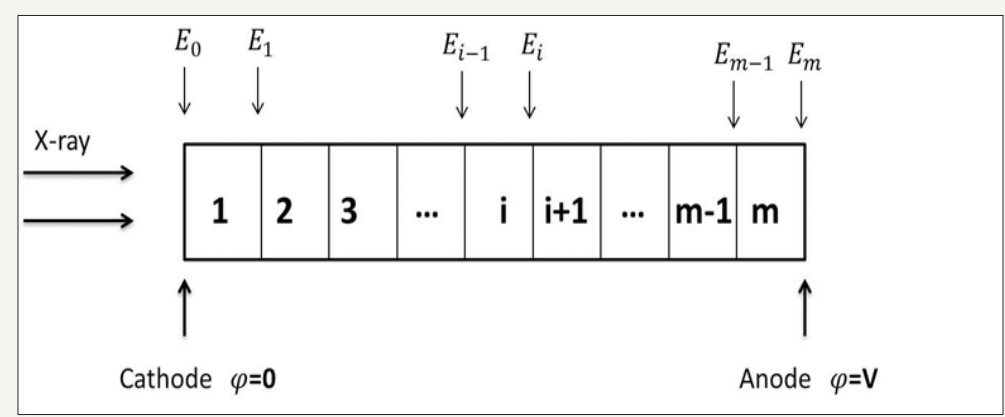

Figure 1: Modeling geometry of the detector.

The corresponding Poisson equation can be rewritten as

$$
\frac{d^{2} \varphi}{d Z^{2}}=-\frac{d E}{d Z}=-\frac{E_{i}-E_{i-1}}{\Delta Z}=\frac{\rho i}{\varepsilon}
$$

where $E_{i}$ is the electric field strength at the $i^{\text {th }}$ and $i+1^{\text {th }}$ cell boundaries. It can be obtained using the boundary conditions given by equation (10).

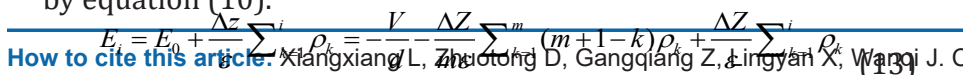

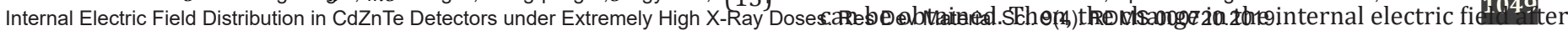

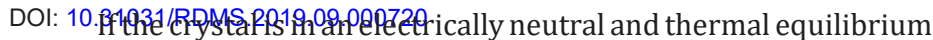
state in the initial state, and the internal electric field is uniform. After time $\Delta \mathrm{t}$, the change in the electron concentration in the $\mathrm{i}^{\text {th }}$ cell state the space charge distribution after every time evolution of $\Delta$

concentration caused by trapping/complexing and the reverse process can be expressed as

$n_{i}\left(1-\exp \left(W_{e t}^{1} \Delta t\right)\right)+n_{t i}^{1}\left(1-\exp \left(W_{e d}^{1} \Delta t\right)\right)+n_{i}\left(1-\exp \left(W_{e t}^{2} \Delta t\right)\right)+n_{t i}^{2}\left(1-\exp \left(W_{e d}^{2} \Delta t\right)\right)$.

By analogy, the change of the hole concentration in the $\mathrm{i}^{\text {th }}$ cell can be obtained due to direct recombination. Based on the initial time $\Delta t$ can be calculated. According to the changed internal electric field, the space charge distribution after the next $\Delta \mathrm{t}$ is calculated. This step is repeated until the steady state is reached. 


\section{Results and Discussion}

It is assumed that the thickness of CdZnTe crystal is $2 \mathrm{~mm}$, the applied external voltage is $500 \mathrm{~V}$, X-ray is incident from the cathode sidess. $\mu_{\mathrm{e}}=1000 \mathrm{~cm}^{2} / \mathrm{Vs}, \mu_{\mathrm{h}}=100 \mathrm{~cm}^{2} /$ Vs and the electron-hole pair ionization energy is $4.7 \mathrm{eV}$. Four representative defect levels are selected in CdZnTe crystal, as shown in Table 1.

Table 1: Defect level parameters.

\begin{tabular}{|c|c|c|c|c|}
\hline Trap & Trap Level/eV & Trap Density $/ \mathrm{cm}^{-3}$ & Capture Cross-Section $/ \mathrm{cm}^{2}$ & Recombination Cross-Section $/ \mathrm{cm}^{2}$ \\
\hline $\mathrm{D}_{1}$ & 1.48 & $5.46 \times 10^{15}$ & $1.0 \times 10^{-19}$ & $3 \times 10^{-14}$ \\
\hline $\mathrm{D}_{2}$ & 0.96 & $3.90 \times 10^{13}$ & $1.2 \times 10^{-16}$ & $3 \times 10^{-14}$ \\
\hline $\mathrm{A}_{1}$ & 0.11 & $4.40 \times 10^{15}$ & $1.54 \times 10^{-18}$ & $3 \times 10^{-14}$ \\
\hline $\mathrm{A}_{2}$ & 0.21 & $1.02 \times 10^{15}$ & $4.83 \times 10^{-18}$ & $3 \times 10^{-14}$ \\
\hline
\end{tabular}

Let X-ray tube voltage, tube current, and action time be $120 \mathrm{kV}, 1.00 \mathrm{~mA}$, and $20 \mathrm{~ms}$, respectively, the carrier concentration distribution is calculated with the results shown in Figure 2.

There is evidence that the trapped hole enriched in the area close to the cathode. As the distance to the anode increases, the number of enriched holes decreases, and the number of electrons increases. Positive space charge regions appear close to the cathode region. The reason on one hand, X-rays are incident from the cathode. On the other hand, owing to the relatively poor transport properties of holes, the mobility of holes is low and easy to be captured. Negative space charge distributes in a wide region and more homogeneous owing to relatively long electron transport distances. The total carrier distribution and internal electric field distribution in the crystal are shown in Figure 3.

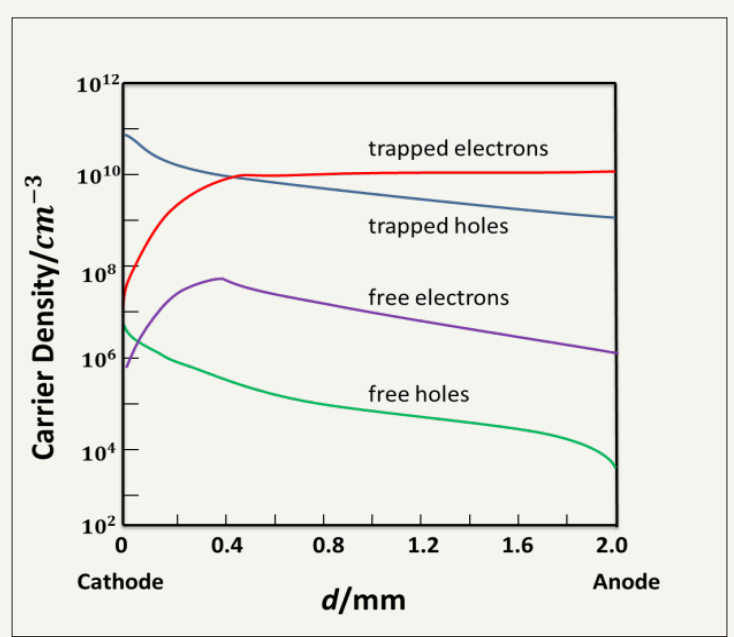

Figure 2: Carrier density distribution (tube voltage: $120 \mathrm{kV}$; tube current: $1.00 \mathrm{~mA}$ ).

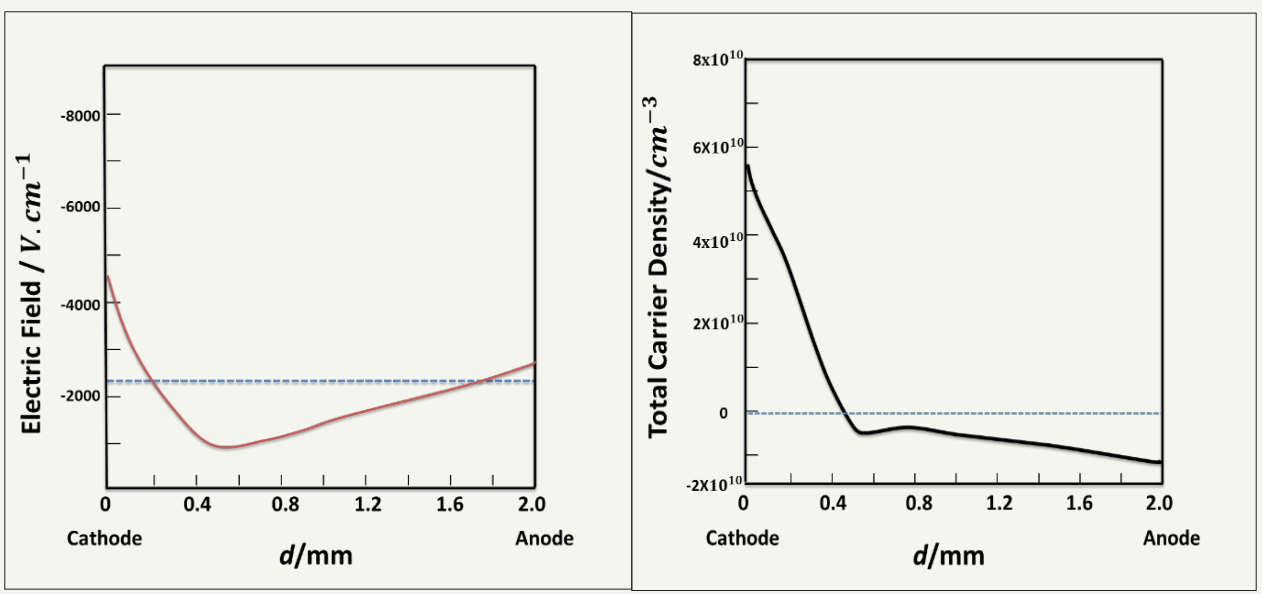

Figure 3: Simulation results at the X-ray tube voltage $120 \mathrm{KV}$ and the tube current $1.00 \mathrm{~mA}$ (a) distribution of electric field and (b) distribution of total carrier density. 
The dotted line indicates the uniform electric field under the action of the external bias. It can be seen from Figure 3(a) that the internal electric field is significantly distorted. The electric field at the cathode has a maximum value and drops rapidly with the increase of the distance from the cathode. After a certain point, it increases again. The internal electric field determines the transit time of electrons in the crystal. The variation of the total charge density is shown in Figure 3(b). High density of the charges is generated near the cathode, which decreases with the increase of the distance. The lower density of the charges in the region away from the cathode is the sum of the native charges and those transported from the region near the cathode. To understand the charge distribution and electric field distribution in the crystal at higher doses, the X-ray tube voltage was set as $120 \mathrm{KV}$ and the tube current was set as $1.60 \mathrm{~mA}$. The results are shown in Figure 4. It is seen that the concentration of the positive space charge accumulated close to the cathode increases with X-ray dose. Accordingly, the charge concentration in the negative space charge region increases, and the distribution of the internal electric field of the crystal changes. In addition, the distortion of the internal point field is more pronounced, particularly at a distance of $0.391 \mathrm{~mm}$ from the cathode, and zero points appear in the internal electric field. This shows that, at this point, the electrostatic force generated owing to the space charge accumulation is equivalent to the electric field force generated in the field of external power. When the electrons generated by a photon are close to the cathode, they are not only affected by the electric force of the external voltage but also by the electrostatic force of the space charge. At the zero point of the electric field, two kinds of electrons are balanced by each other. After the zero point toward the anode, the externally biased electric field force plays the dominant role and the magnitude of the internal electric field recovers gradually. Under such an electric field distribution, the transport of electrons in the crystal is more severely hindered and the transit time is considerably increased, which leads to further deterioration in the detector's counting performance.
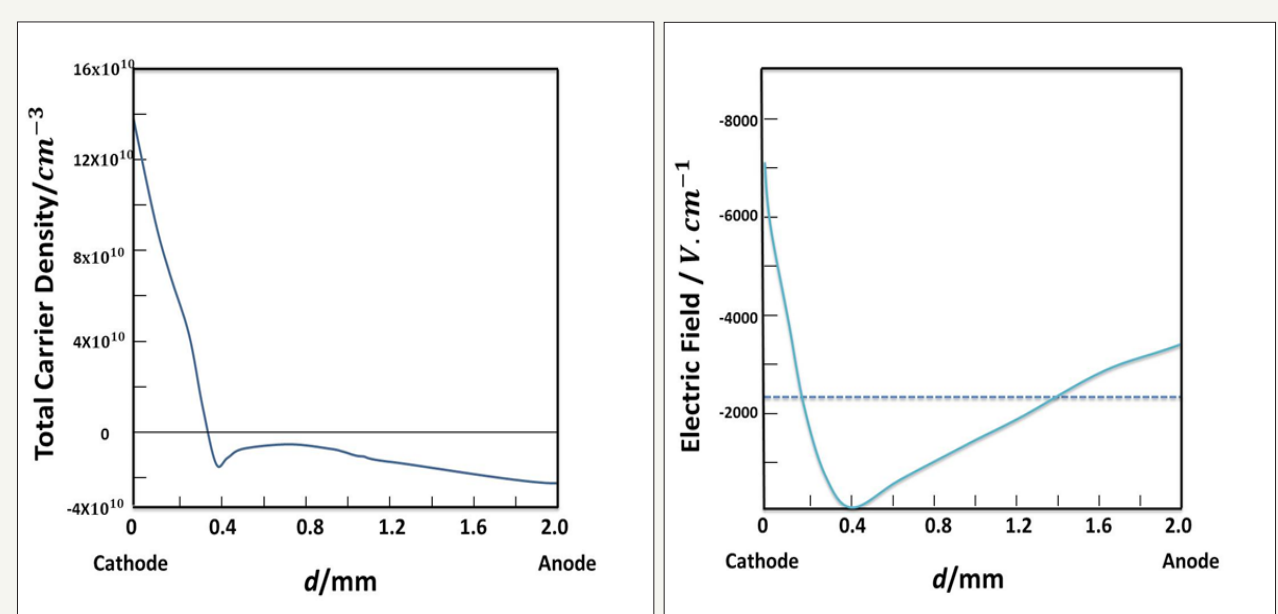

Figure 4: Simulation results at the X-ray tube voltage $120 \mathrm{KV}$ and the tube current $1.60 \mathrm{~mA}$ (a) distribution of total carrier density and (b) inner electric field.
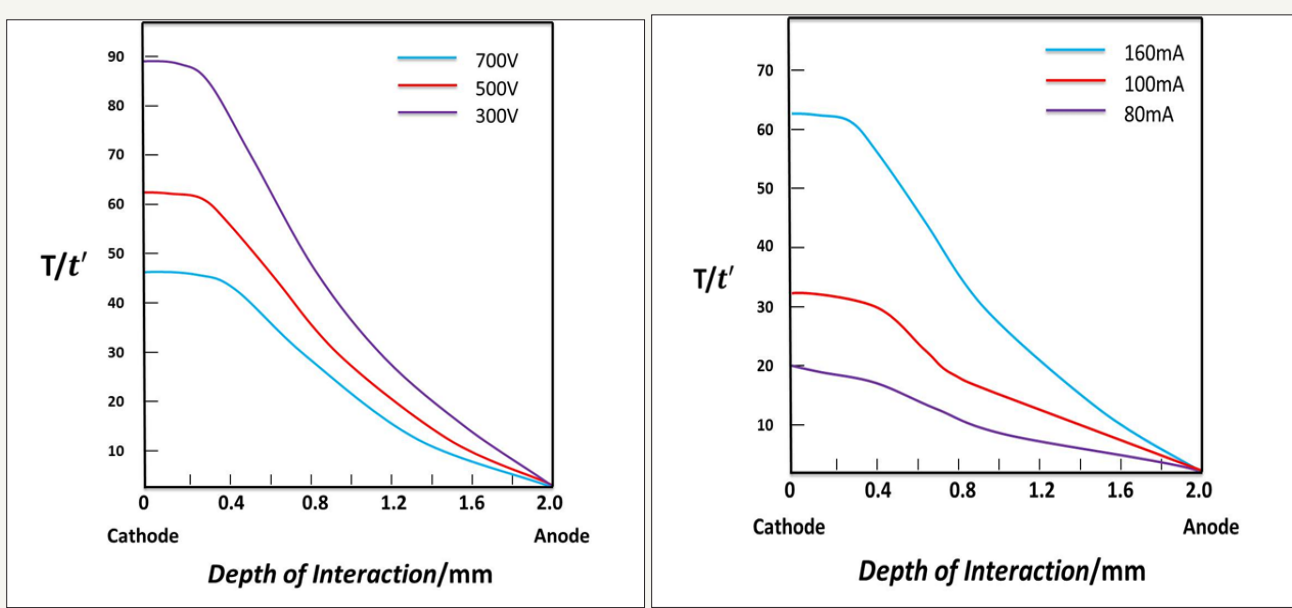

Figure 5: Variation in relative transit time of electrons with the depth of interaction

(a) at different bias voltage, (b) at different X-ray flux. 
Figure 5(a) shows the variation in the relative transit time of electrons in the crystal at different doses of lower radiation, where $\mathrm{t}^{\prime}=\mathrm{L}^{2} / \mu_{\mathrm{e}} \mathrm{V}$, that is, the transit time under the uniform electric field generated by the external bias. The X-ray tube voltage is $120 \mathrm{kV}$, and the external bias is $500 \mathrm{~V}$. Owing to the presence of a positive space charge region close to the cathode, the transit time of the electrons is significantly increased. The accumulated space charge concentration and the transit time of the electrons generated at the same position increase with X-ray dose. Larger transit times indicate an increased likelihood of trapping, which leads to reduced detector performance. Figure 5(b) shows the variation in the relative transit time of electrons in the crystal under different external bias voltages. Increasing external bias decreases the transit time of electrons, thereby reduces the probability of capture and recombination and improves collection efficiency. However, the leakage current and noise increase with the external voltage. Therefore, the external bias voltage is selected according to the resistivity of wafers.

\section{Conclusion}

The carrier distribution, space charge accumulation, and internal electric field distribution in CdZnTe crystals were calculated with consideration of four kinds of defect traps under extremely high X-ray doses. It was revealed that under extremely high doses of radiation, space charge accumulated in the region close to the cathode. This affected the distribution of the internal electric field and thus the performance of the detector. Electron transit time increased with X-ray dose ansssd applied bias voltage.

\section{Acknowledgment}

This work was financially supported by National Natural Science Foundations of China (Nos. 51502244, 51702271, U1631116 and 51372205), National Key Research and Development Program of China (2016YFF0101301 and 2016YFE0115200), and the fund of the State Key Laboratory of Solidification Processing in NWPU (SKLSP201741). It was also supported by the Natural Science Basic Research Plan in Shaanxi Province of China (2016KJXX-09) and the Fundamental Research Funds for the Central Universities (3102015BJ(II)ZS014).

\section{References}

1. Shikhaliev PM, Fritz SG (2011) Photon counting spectral CT versus conventional CT: comparative evaluation for breast imaging application. Phys Med Biol 56(7): 1905-1930.

2. Schlesinger, Toney JE, Yoon H, Lee EY (2001) Cadmium zinc telluride and its use as a nuclear radiation detector material. Materials Science \& Engineering R 32(4): 103-189.

3. Del SS, Leonardo A, Ezio C, Maria MA, Andrea Z, et al. (2009) Progress in the development of CdTe and CdZnTe semiconductor radiation detectors for astrophysical and medical applications. Sensors 9(5): 3491-3526.
4. Abbene L, Gerardi G (2011) Performance enhancements of compound semiconductor radiation detectors using digital pulse processing techniques. Nuclear Inst \& Methods in Physics Research A 654(1): 340348 .

5. Taguchi K, Iwanczyk JS (2013) Vision 20/20: Single photon counting $\mathrm{x}$-ray detectors in medical imaging. Med Phys 40(10): 100901.

6. Kurková D, L Judas (2016) X-ray tube spectra measurement and correction using a CdTe detector and an analytic response matrix for photon energies up to $160 \mathrm{keV}$. Radiation Measurements 85: 64-72.

7. Strassburg M, Schroeter C, Hackenschmied P (2011) CdTe/CZT under high flux irradiation. Journal of Instrumentation 6(1): C01055.

8. Bale DS, Szeles C, (2008) Nature of polarization in wide-bandgap semiconductor detectors under high-flux irradiation: Application to semi-insulating Cd (1 - x) Zn(X)Te. Phys rev B 77(3): 035205-035205.

9. Engel KJ, Herrmann C (2011) Simulation of one-dimensionally polarized $\mathrm{x}$-ray semiconductor detectors. Proceedings of SPIE - The International Society for Optical Engineering 7961(2): 126-128.

10. Gao W, Li X, Liu H, Hu Y, (2016) Design and performance of a 16-channel radiation-hardened low-noise front-end readout ASIC for CZT-based hard X-ray imager. Microelectronics Journal 48(C): 87-94.

11. Gao W, Gan B, Li X, Wei T, Gao D, et al. (2015) Development of a compact radiation-hardened low-noise front-end readout ASIC for CZT-based hard X-ray imager. Nuclear Inst \& Methods in Physics Research A 780: $15-20$

12. Bale DS, Soldner SA, Szeles C (2008) A mechanism for dynamic lateral polarization in CdZnTe under high flux x-ray irradiation. Applied Physics Letters 92(8): 035205.

13. Soldner SA, Bale DS, Szeles C (2007) Dynamic lateral polarization in cdznte under high flux x-ray irradiation. IEEE Transactions on Nuclear Science 54(5): 1723-1727.

14. Camarda GS, Bolotnikov AE, Cui Y, Hossain A, Awadalla SA, et al. (2009) Polarization studies of cdznte detectors using synchrotron x-ray radiation. IEEE Transactions on Nuclear Science 55(6): 3725-3730.

15. Pautrat JL, Francou JM, Magnea N, Molva E, Saminadayar K (1985) Donors and acceptors in tellurium compounds; The problem of doping and self-compensation. Journal of Crystal Growth 72(1): 194-204.

16. Franc J, Dědič V, Rejhon M, Zázvorka J, Praus P, et al. (2015) Control of electric field in CdZnTe radiation detectors by above-bandgap light. Journal of Applied Physics 117(16): 507

17. Xu L, Jie W, Zha G, Feng T, Wang N, et al. (2014) Effects of sub-bandgap illumination on electrical properties and detector performances of CdZnTe. Applied Physics Letters 104(23): 103.

18. Alekseeva L, Dorogov P, Ivanov V, Loutchanski A (2011) Investigation of the influence of light illumination on the characteristics of CdZnTe detectors. Nuclear Science Symposium and Medical Imaging Conference, pp. 4562-4566

19. Prokesch M, Szeles C (2006) Accurate measurement of electrical bulk resistivity and surface leakage of CdZnTe radiation detector crystals. Journal of Applied Physics 100(1): 103.

20. Le HQ DucoteJL, Molloi S (2010) Radiation dose reduction using a Cd ZnTe-based computed tomography system: comparison to flat-panel detectors. Med Phys 37(3): 1225-1236. 
Creative Commons Attribution 4.0

International License

For possible submissions Click Here

\section{Research \& Development in Material Science}

\section{Benefits of Publishing with us}

- High-level peer review and editorial services

- Freely accessible online immediately upon publication

- Authors retain the copyright to their work

- Licensing it under a Creative Commons license

- Visibility through different online platforms 